\title{
Manufacturing and morphing behavior of high-amplitude corrugated laminates
}

\section{Other Conference Item}

Author(s):

Kress, Gerald; Filipovic, Daniel

Publication date:

2019-06-16

Permanent link:

https://doi.org/10.3929/ethz-b-000350014

Rights / license:

In Copyright - Non-Commercial Use Permitted

Funding acknowledgement:

169468 - Structural Response and Manufacturing of Corrugated Laminates (SNF) 


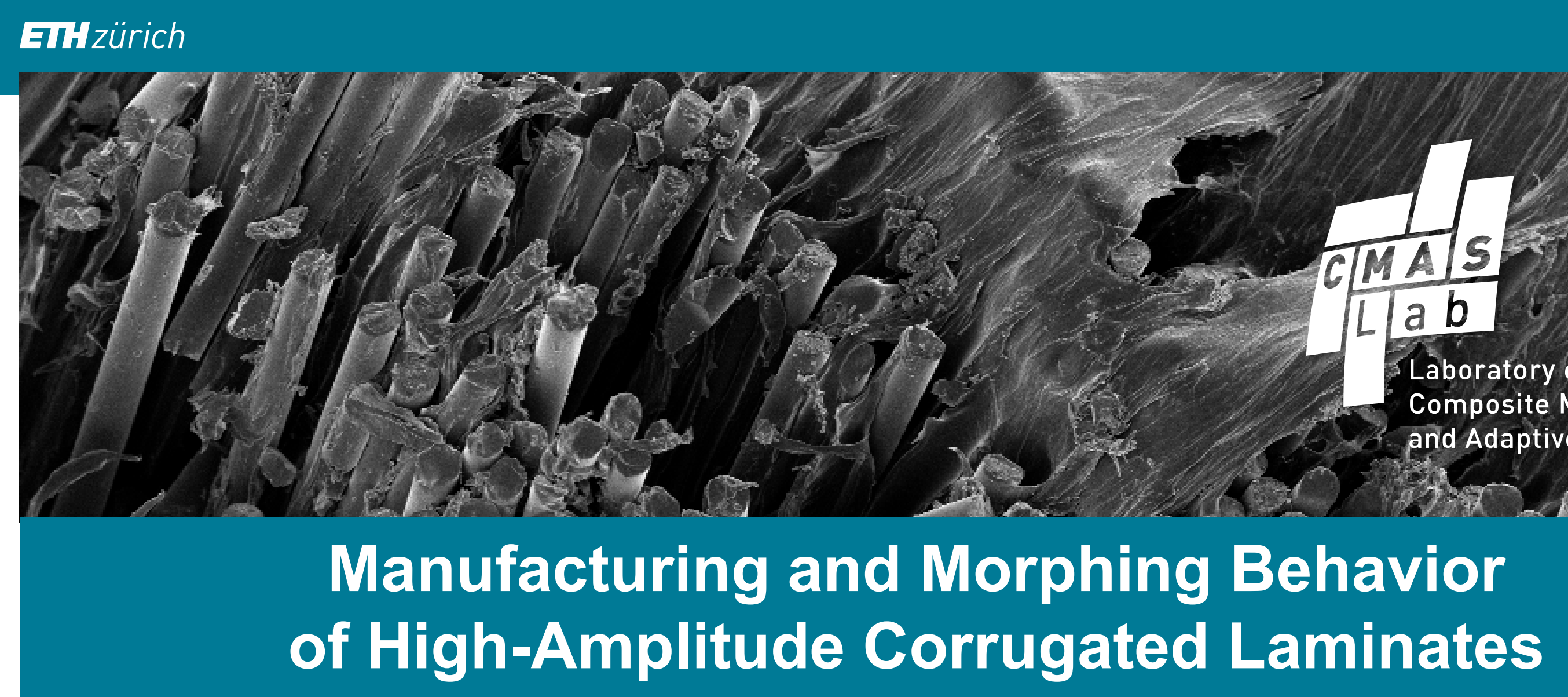

Gerald Kress, Daniel Filipovic

ICoNSoM, Rome, June 16-19, 2019 


\section{Contents}

- Extreme anisotropy of corrugated laminates

- Flexible skins in morphing-wing design

- Arguments for high-amplitude circular-sections shape

- Manufacturing of high-amplitude corrugated laminates

- Mathematical background for design

- Manufacturing and prototype

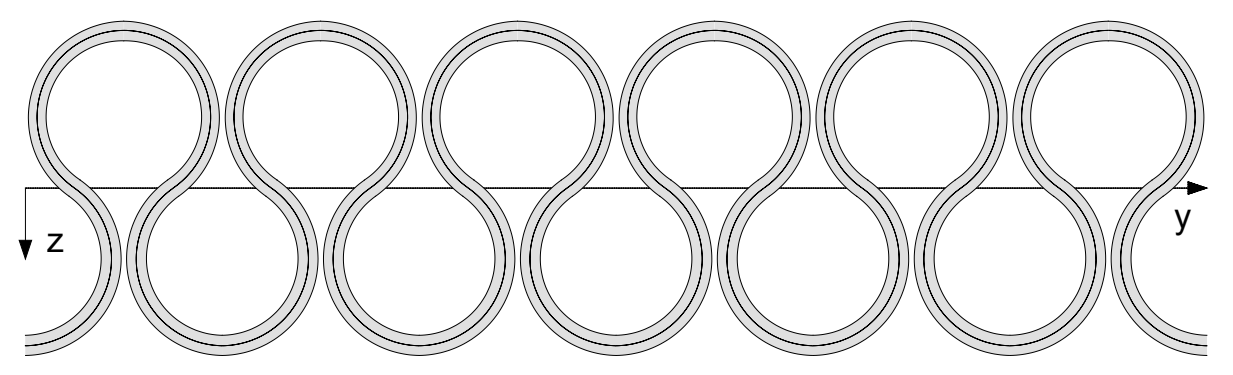

- Morphing model

- Theory

- Results and verification

- Conclusion and outlook 
Extreme anisotropy of high-amplitude corrugated laminates
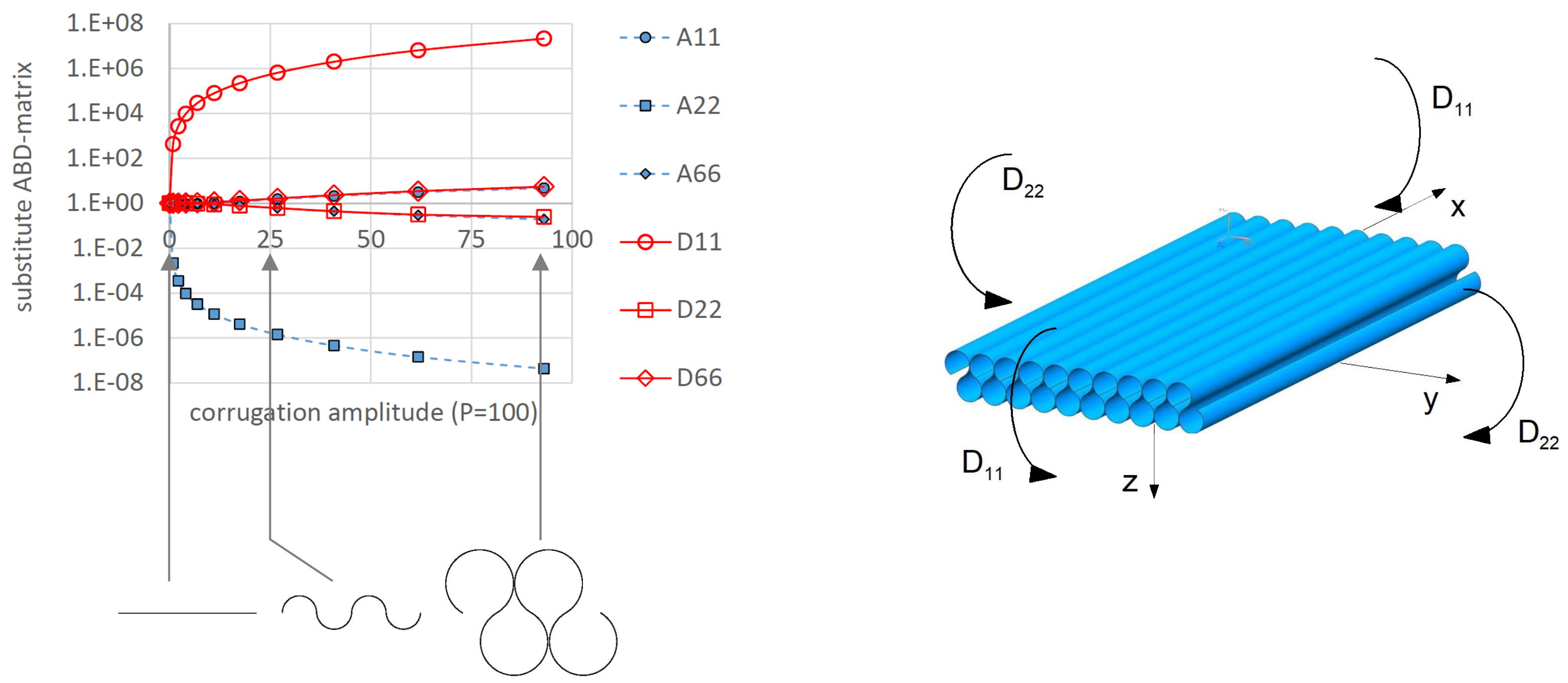


\section{Arguments for use of corrugated laminates as flexible skin in morphing-wing design}

- High in-plane stiffness $A_{11}$ contributes to wing-skin span-wise stiffness

- Low in-plane stiffness $A_{22}$ allows for large chord-wise stretch

$\circ$ Very high bending stiffness $D_{11}$ adds the function of local stringer

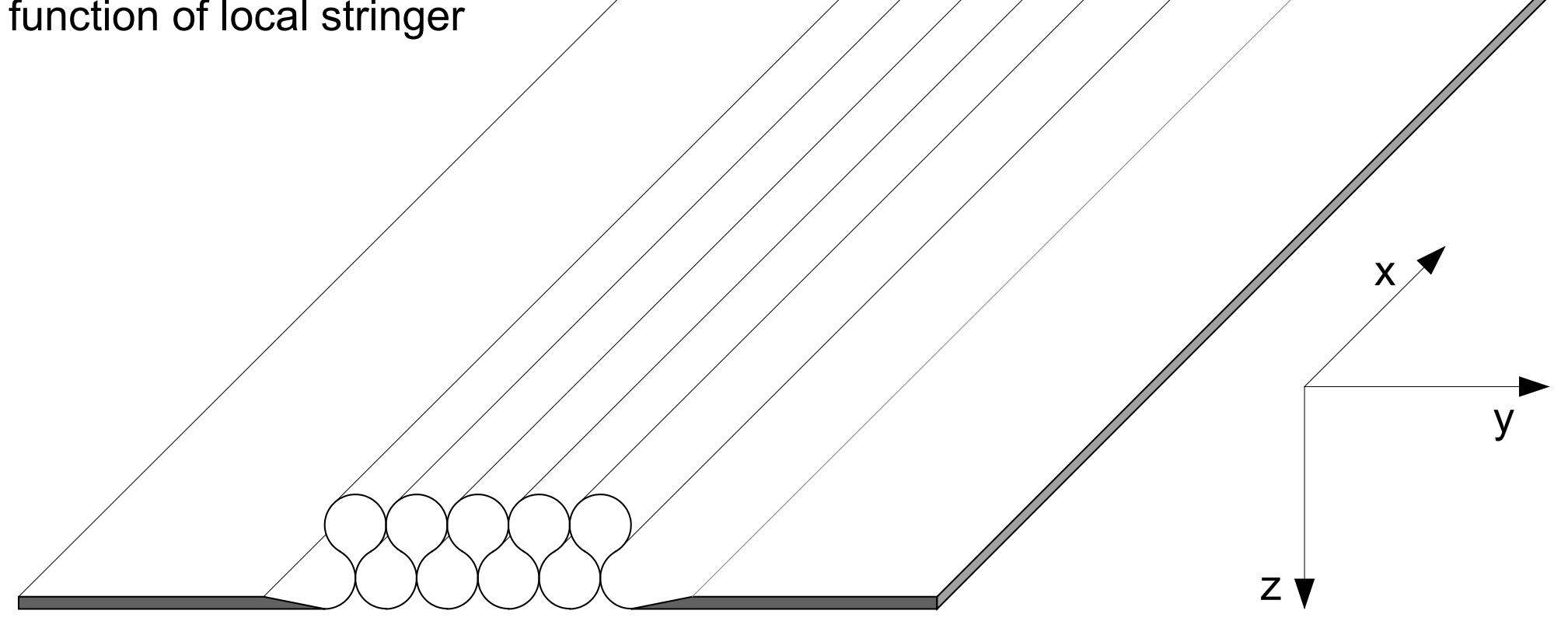


Viable method for realizing high-amplitude corrugated circular-sections-shape laminates

- Because of undercuts it is difficult to imagine that use of molds could lead to economic manufacturing process

- Forming of laminates by thermal effects has already been pointed out in the literature $[3,4]$

- The suggestion is applied to manufacture of corrugated laminates 


\section{ヨHzürich}

Circular-segment corrugation shape conventions

Periodic length $P$ and corrugation amplitude $c$ determine curvature radius $R$ :

$R=\frac{16 c^{2}+P^{2}}{32 c}$

Dimensionless geometry values:

$\bar{c}=\frac{c}{P}, \bar{t}=\frac{t}{P}, \quad \bar{R}=\frac{R}{P}, \quad \bar{P}=1$

Characteristic opening angle: $\quad \psi_{0}=\arccos \left(1-\frac{c}{R}\right)$

Dimensionless curved length: $\quad \bar{L}_{s}=4 \psi_{0} \bar{R}$ 
Manufacturing and morphing-performance performance arguments for high-amplitude shape

Shape curvature is smaller for highest than for intermediate amplitudes:

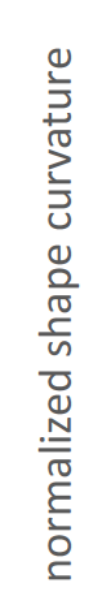

Stretch values by unbending action increase significantly with corrugation amplitude:

$$
\lambda=\bar{L}_{s}
$$

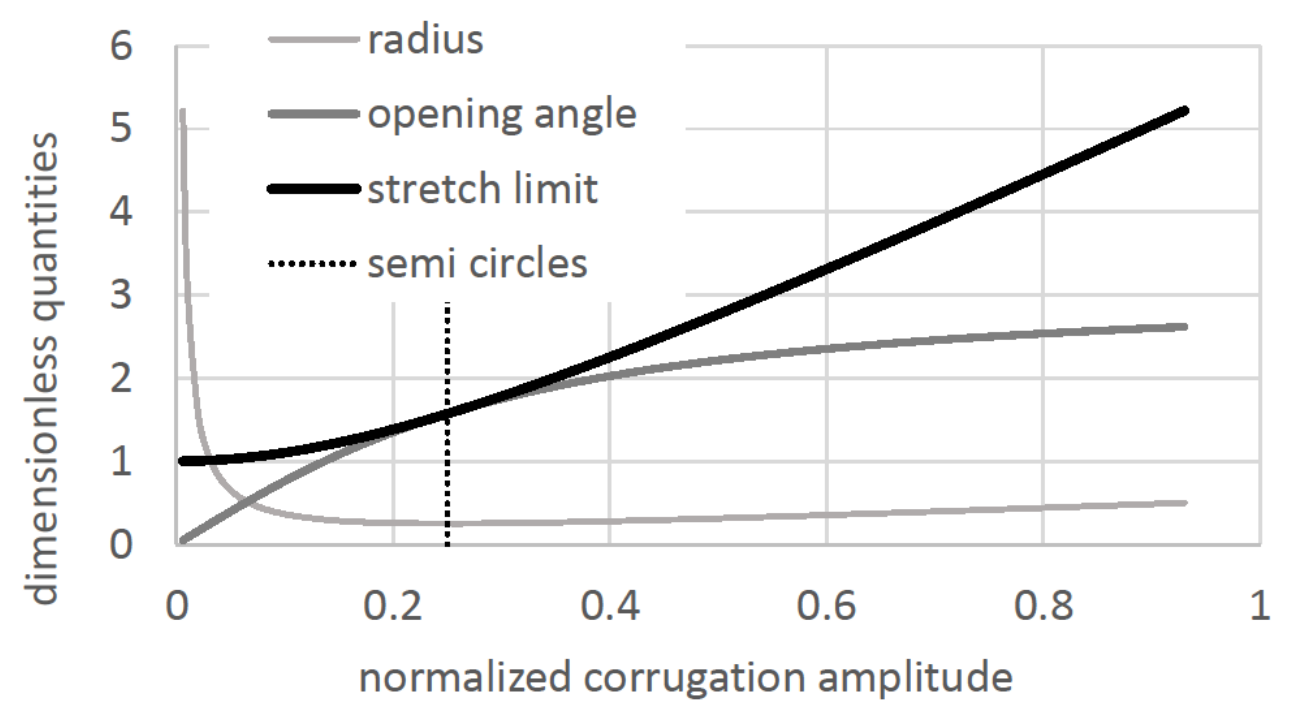




\section{Recall of classical theory of laminated plates}

$\left\{\begin{array}{l}\mathbf{N} \\ \mathbf{M}\end{array}\right\}^{\text {equ }}=\sum_{k=1}^{N} \overline{\mathbf{Q}}_{k}\left(\overline{\boldsymbol{\alpha}}_{k} \Delta T+\overline{\boldsymbol{\beta}}_{k} \Delta H\right)\left\{\begin{array}{r}\left(z_{k}-z_{k-1}\right) \\ \frac{1}{2}\left(z_{k}^{2}-z_{k-1}^{2}\right)\end{array}\right\} \quad\left\{\begin{array}{l}\mathbf{N} \\ \mathbf{M}\end{array}\right\}^{\text {fict }}=\left\{\begin{array}{l}\mathbf{N} \\ \mathbf{M}\end{array}\right\}^{\text {equ }}+\left\{\begin{array}{l}\mathbf{N} \\ \mathbf{M}\end{array}\right\}^{\text {mec }}=\left[\begin{array}{ll}\mathbf{A} & \mathbf{B} \\ \mathbf{B} & \mathbf{D}\end{array}\right]\left\{\begin{array}{l}\boldsymbol{\varepsilon}^{0} \\ \mathbf{\kappa}\end{array}\right\}$

Realistic reduced system for estimating thermal laminate curvature under constraint of cylindrical bending:

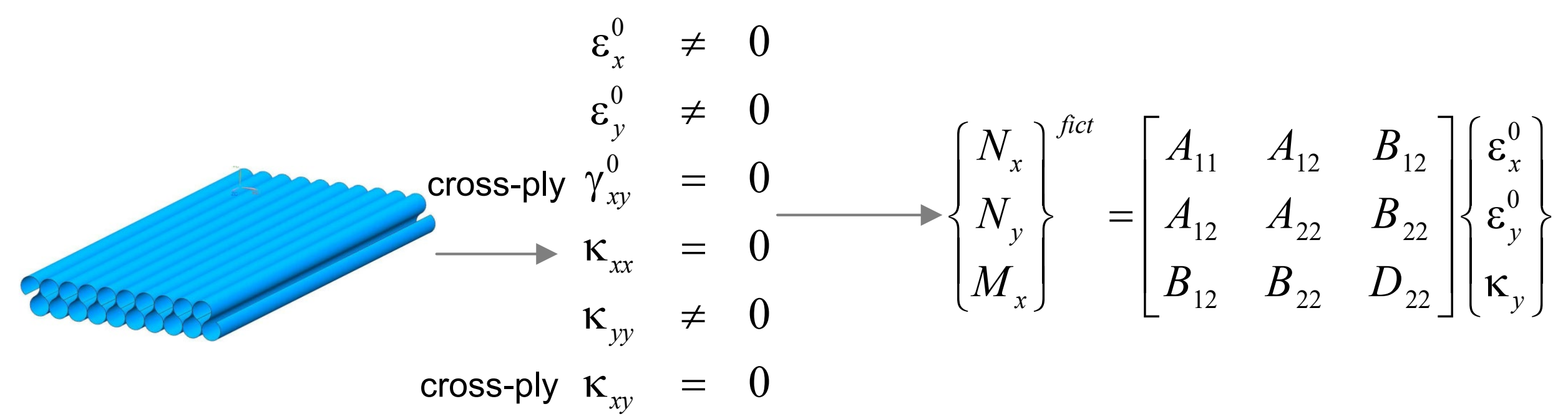




\section{Material data and thermally induced curvatures for cylindrical bending}

Table 1: Relevant material properties of unidirectional composites with fiber-volume fraction $v_{f}=0.6$. Source: DORNIER SYSTEM $\mathrm{GmbH}$

\begin{tabular}{l|c|c|c|c|c} 
fiber & $E_{1}$ & $E_{2}$ & $v_{12}$ & $\alpha_{1}$ & $\alpha_{2}$ \\
\hline & \multicolumn{2}{|c}{$[\mathrm{MPa}]$} & {$[-]$} & \multicolumn{2}{c}{$\left[10^{-6} / K\right]$} \\
\hline T 300 & 135000 & 10000 & 0.27 & -0.6 & 30 \\
M 40 & 220000 & 7000 & 0.35 & -0.8 & 30 \\
GY 70 & 290000 & 5000 & 0.41 & -1.0 & 30
\end{tabular}

\section{Conclusion:}

Material system with moderately stiff fibers provides highest thermal curvatures in $\left[0 / 90_{2}\right]$ or $\left[90_{2} / 0\right]$ laminates
Table 2: Temperature-curvature coefficients $\kappa_{y}^{T}\left[10^{-4} / \mathrm{mmK}\right]$

\begin{tabular}{l|c|c|c|c} 
fiber & {$[90 / 0]$} & {$\left[90 / 0_{2}\right]$} & {$\left[90 / 0_{3}\right]$} & {$\left[90 / 0_{4}\right]$} \\
\hline T 300 & 1.0233 & 1.0863 & 0.8863 & 0.7149 \\
M 40 & 0.6348 & 0.9081 & 0.8395 & 0.7148 \\
GY 70 & 0.4066 & 0.7214 & 0.7571 & 0.6848
\end{tabular}

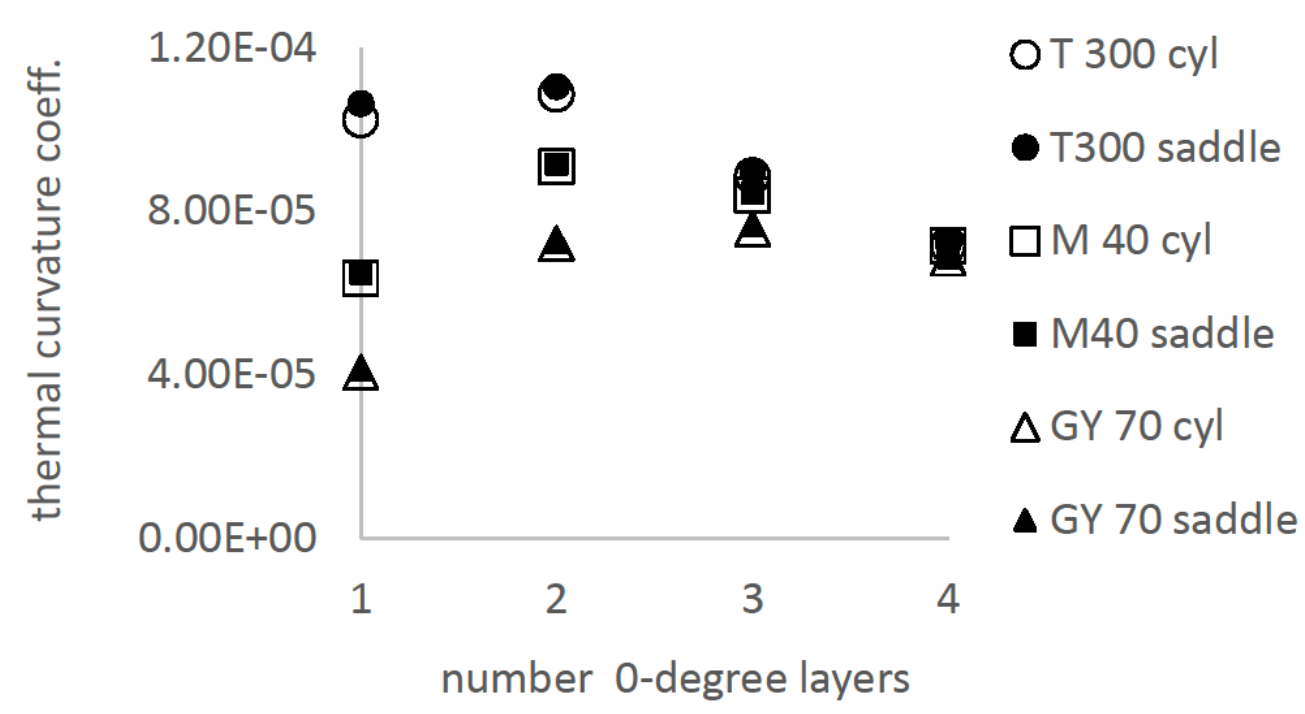




\section{Manufacturing: Prototype}

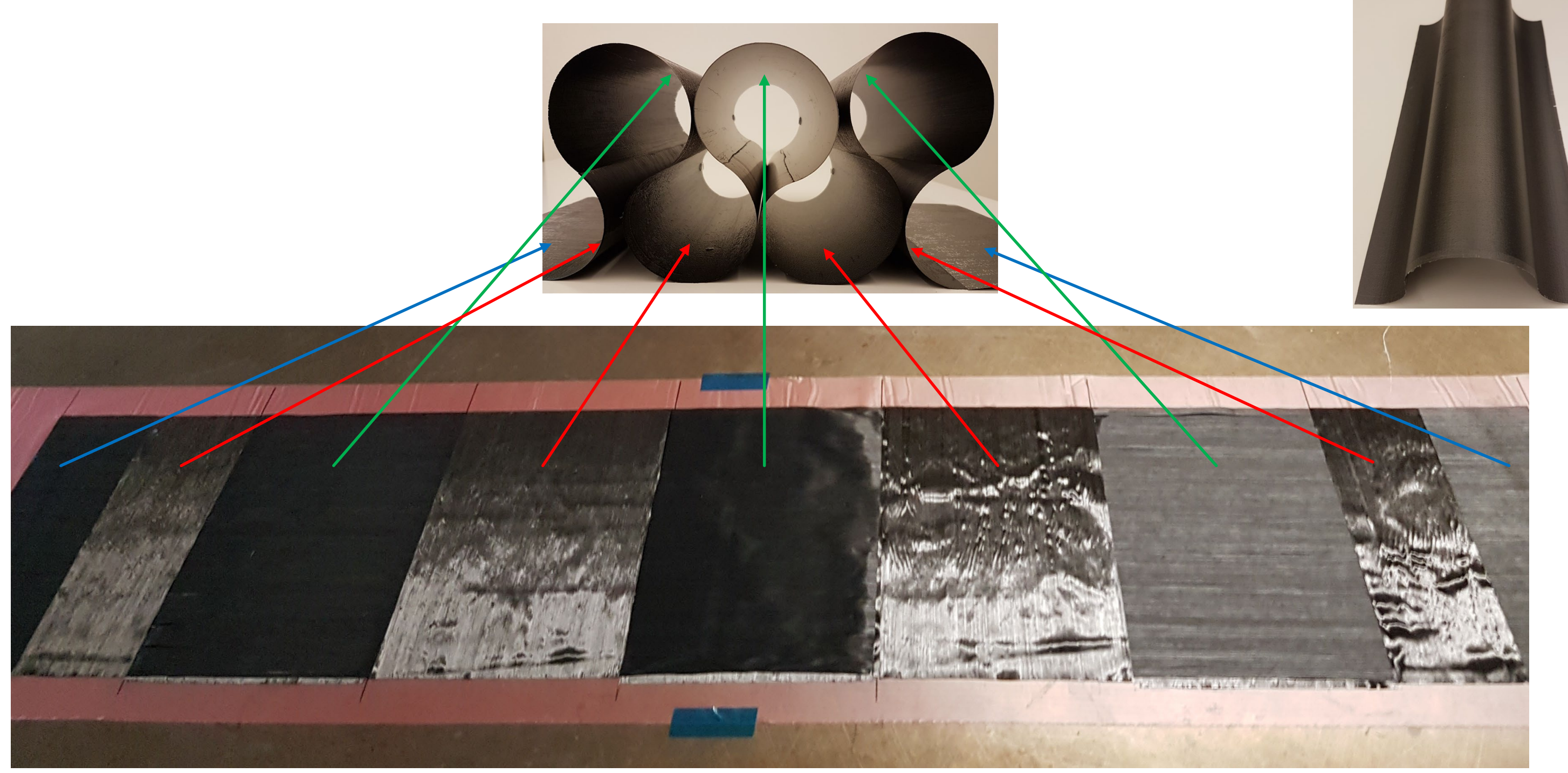


Conclusion and outlook manufacturing part:

- circular-sections corrugated laminates can be realized in good quality and moderate manufacturing effort by using

- unsymmetric cross-ply laminates

- elevated curing temperatures

- however, laminate-thickness to curvature-radius ratios remain small

- More details on mathematical estimate of curvature radius and other aspects are found in [5]

- Morphing models must take into account unsymmetric laminate design ! 
Conclusion and outlook manufacturing part: Aerodynamically smooth surface
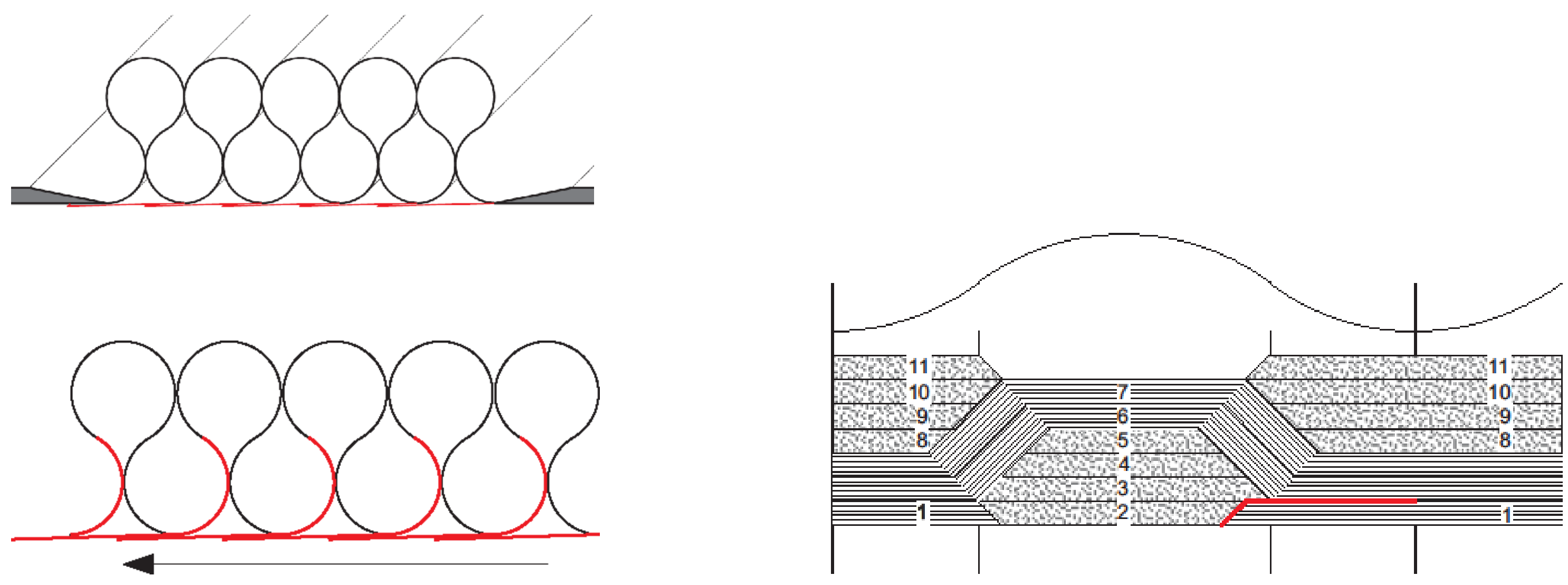
Nonlinear morphing model: Geometric and mechanical unit cells
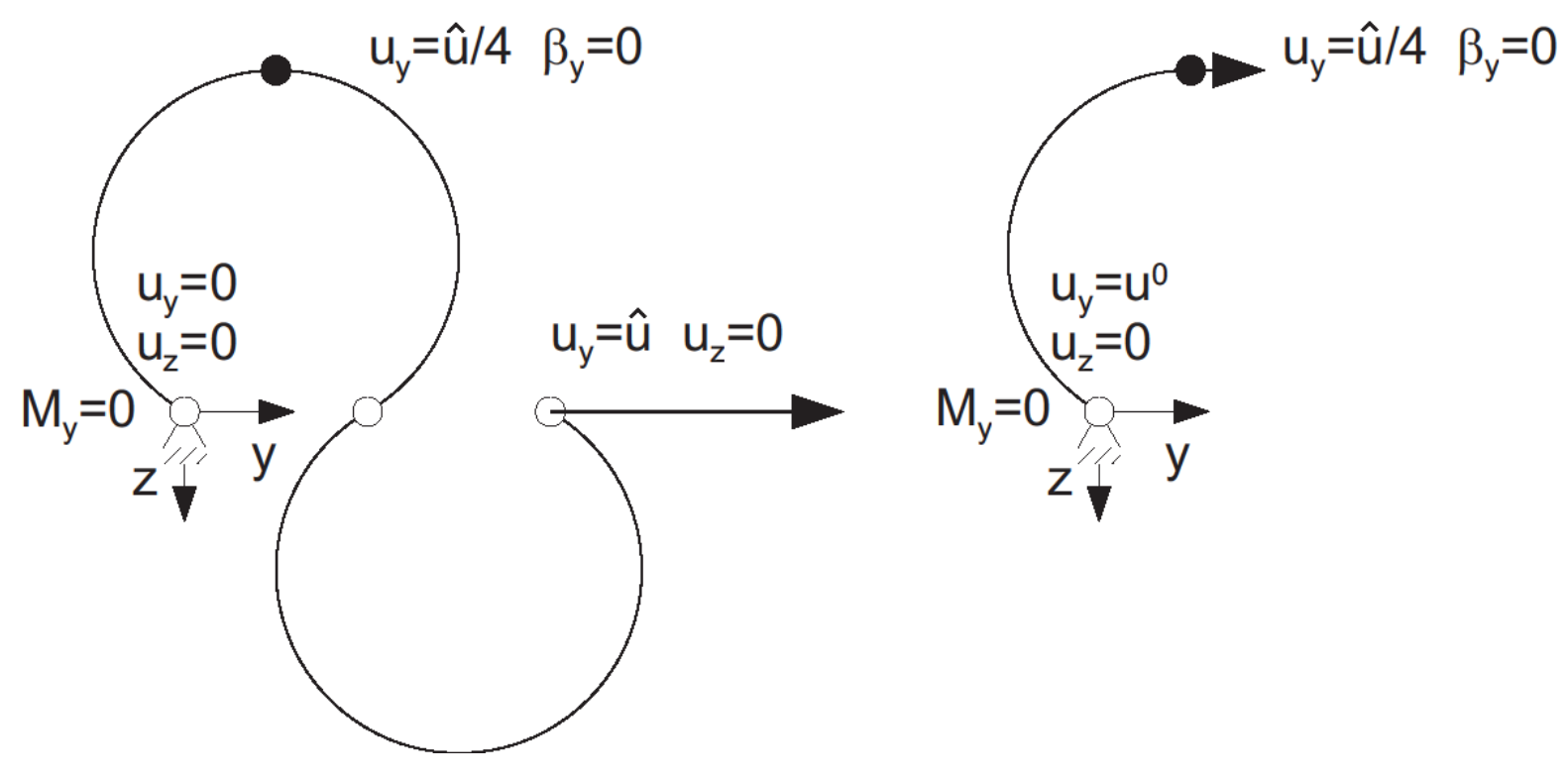

Geometric periodic unit cell:

- statically determinate support

- periodicity conditions
Mechanical unit cell:

existing symmetries justify quarter unit-cell model

- simply supported at origin

- rotation: homogeneous EBC

- displacement: finite values specified 


\section{캐zürich}

Nonlinear morphing model: Coordinate transformation and reference configuration

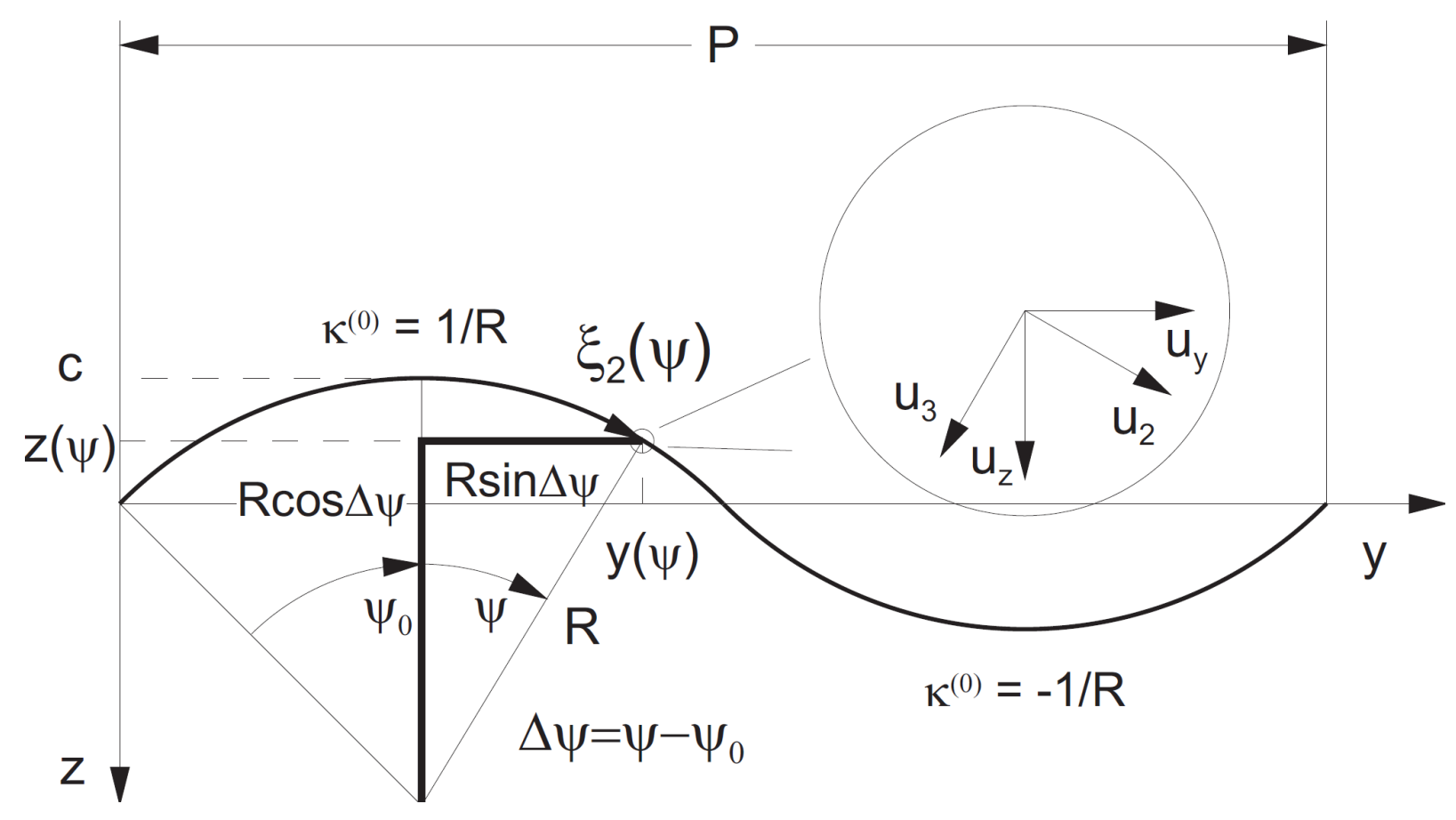

$$
\begin{aligned}
& y^{(0)}\left(\xi_{2}\right)=R \sin \Delta \psi \\
& z^{(0)}\left(\xi_{2}\right)=R(1-\cos \Delta \psi)-c
\end{aligned}
$$$$
\Delta \psi=\psi-\psi_{0}=\frac{\xi_{2}}{R}-\psi_{0}=\kappa^{0} \xi_{2}-\psi_{0}
$$

coordinate transformation

$$
\left\{\begin{array}{l}
x \\
\xi_{2} \\
\xi_{3}
\end{array}\right\}=\left[\begin{array}{ccc}
1 & 0 & 0 \\
0 & \cos \theta & \sin \theta \\
0 & -\sin \theta & \cos \theta
\end{array}\right]\left\{\begin{array}{l}
x \\
y \\
z
\end{array}\right\}
$$




\section{캐zürich}

Nonlinear morphing model: Cylindrical bending, constitutive equations, external equilibrium reference configuration curvature : $\kappa$ bending curvature $: \varepsilon^{1}$

$N_{x}=A_{12} \varepsilon_{2}^{0}$

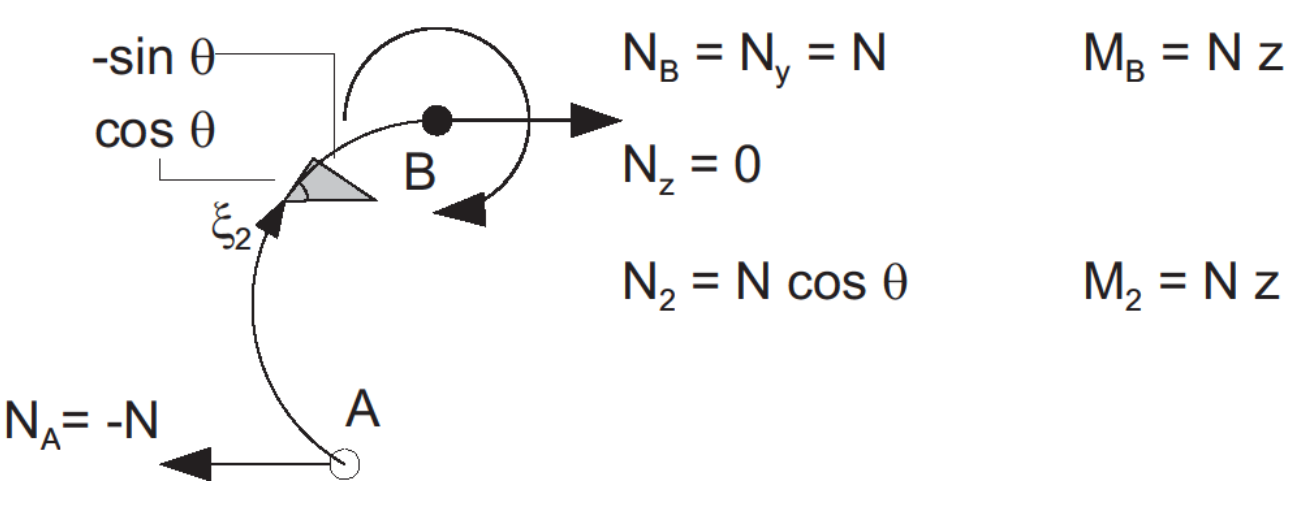

$N_{2}=A_{22} \varepsilon_{2}^{0}+B_{22} \varepsilon_{2}^{1}$

$M_{x}=\quad D_{12} \varepsilon_{2}^{1}$

$M_{2}=B_{22} \varepsilon_{2}^{0}+D_{22} \varepsilon_{2}^{1}$

$$
\begin{aligned}
& \left\{\begin{array}{c}
N \cos \theta \\
N z
\end{array}\right\}=\left[\begin{array}{ll}
A_{22} & B_{22} \\
B_{22} & D_{22}
\end{array}\right]\left\{\begin{array}{c}
\varepsilon_{2}^{0} \\
\varepsilon_{2}^{1}
\end{array}\right\} \\
& \left\{\begin{array}{c}
\varepsilon_{2}^{0} \\
\varepsilon_{2}^{1}
\end{array}\right\}=\frac{1}{\operatorname{det}}\left[\begin{array}{rr}
D_{22} & -B_{22} \\
-B_{22} & A_{22}
\end{array}\right]\left\{\begin{array}{c}
N \cos \theta \\
N z
\end{array}\right\} ; \operatorname{det}=A_{22} D_{22}-B_{22}^{2}
\end{aligned}
$$




\section{Nonlinear morphing model: Deformed configuration}

Total curvature is the sum of

shape and bending curvatures:

$\kappa^{(t)}\left(\xi_{2}\right)=\kappa^{(0)}+\varepsilon_{2}^{1}\left(\xi_{2}\right)$

Cross-sectional rotation follows from integrating the total curvature:

$\theta^{(t)}\left(\xi_{2}\right)=\int_{0}^{\xi_{2}} \kappa^{(t)}(\sigma) d \sigma+C_{1}$

Stretch of line elements [7]:

$d \xi_{2}^{(t)}=\lambda d \xi_{2}^{(0)}=\left(1+\varepsilon_{2}^{0}\right) d \xi_{2}^{(0)}$
Incremental displacements due to stretch [7]:

$$
d u_{y}^{0}=\cos (\theta) \varepsilon_{2}^{0} d \xi_{2}^{(0)} ; \quad d u_{z}^{0}=-\sin (\theta) \varepsilon_{2}^{0} d \xi_{2}^{(0)}
$$

Incremental displacements due to stretch:

$$
d u_{y}=d u_{y}^{0}+d u_{y}^{1} ; \quad d u_{z}=d u_{z}^{0}+d u_{z}^{1}
$$

\section{Deformed configuration:}

$$
\begin{aligned}
& y^{(t)}=\int_{0}^{\xi_{2}} \cos (\theta) \lambda d \sigma \quad z^{(t)}=-\int_{0}^{\xi_{2}} \sin (\theta) \lambda d \sigma \\
& y_{B}^{(t)}=\int_{0}^{L_{s}} \cos (\theta) \lambda d \sigma \quad z_{B}^{(t)}=-\int_{0}^{L_{s}} \sin (\theta) \lambda d \sigma
\end{aligned}
$$




\section{Nonlinear morphing model: Solution algorithm}

It is desired to specifiy global morphing stretch

$$
x^{(0)}, N=0, \hat{\mathbf{u}}=0
$$

$\rightarrow \quad$ outer process

However, deformed configurations depend most directly on applied line force $\mathrm{N}$

$\rightarrow \quad$ inner process

Loop on load steps creates data points for force-displacement diagrams

Outer process then finds force corresponding with specified maximum stretch

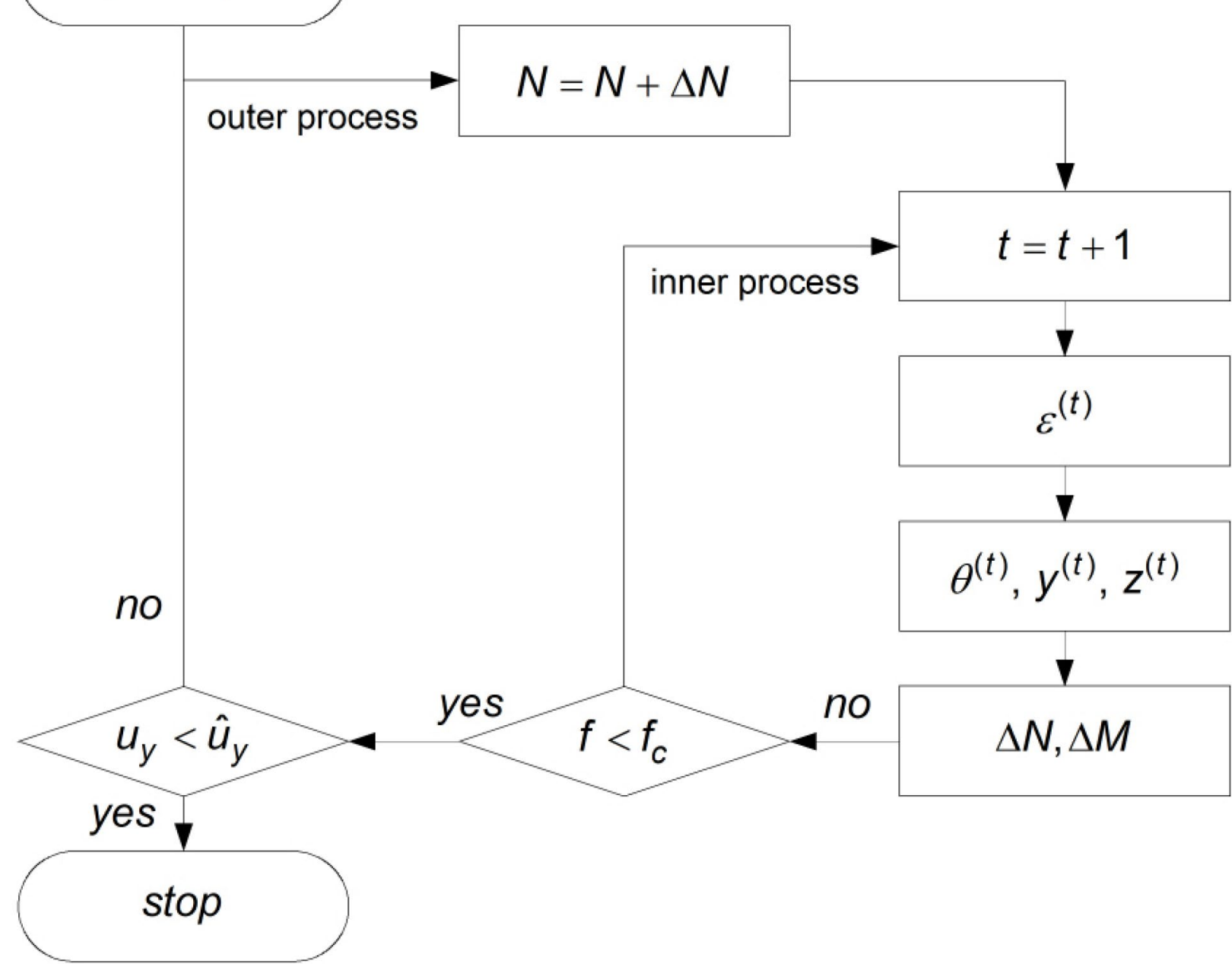




\section{Nonlinear morphing model: Solution algorithm inner process}

Minimize scalar error measure $f$ :

$$
f=\int_{0}^{L_{s}}\left\{\begin{array}{l}
\Delta N_{2} \\
\Delta M_{2}
\end{array}\right\}^{T}\left\{\begin{array}{l}
\Delta N_{2} \\
\Delta M_{2}
\end{array}\right\} d s
$$

$\left\{\begin{array}{l}\Delta N_{2} \\ \Delta M_{2}\end{array}\right\}=\left\{\begin{array}{l}\Delta N_{2}^{\text {int }} \\ \Delta M_{2}^{\text {int }}\end{array}\right\}-\left\{\begin{array}{l}\Delta N_{2}^{\text {ext }} \\ \Delta M_{2}^{\text {ext }}\end{array}\right\}$

$\left\{\begin{array}{l}\Delta N_{2}^{\mathrm{int}} \\ \Delta M_{2}^{\text {int }}\end{array}\right\}=\left[\begin{array}{ll}A_{22} & B_{22} \\ B_{22} & D_{22}\end{array}\right]\left\{\begin{array}{l}\varepsilon_{2}^{0} \\ \varepsilon_{2}^{1}\end{array}\right\}$

Loads from external equlibrium
Numerical version:

$$
f=\sum_{k=1}^{n+1} f_{k}=\sum_{k=1}^{n+1}\left\{\begin{array}{l}
\Delta N_{2} \\
\Delta M_{2}
\end{array}\right\}_{k}^{T}\left\{\begin{array}{l}
\Delta N_{2} \\
\Delta M_{2}
\end{array}\right\}_{k}
$$

The laminate deformations $\varepsilon^{0}{ }_{2 \mathrm{k}}$ and $\varepsilon^{1}{ }_{2 \mathrm{k}}$ control the deformed configuration and are solution parameters.

Error minimization after Newton optimization method:

$$
\begin{aligned}
& \boldsymbol{\varepsilon}_{k}^{(t+1)}=\boldsymbol{\varepsilon}_{k}^{(t)}+\Delta \boldsymbol{\varepsilon}_{k}^{(t)} \quad \boldsymbol{\varepsilon}_{k}^{(t)}=\left\{\begin{array}{c}
\varepsilon_{2}^{0} \\
\varepsilon_{2}^{1}
\end{array}\right\}_{k}^{(t)} \quad \Delta \boldsymbol{\varepsilon}_{k}^{(t)}=-(\nabla \nabla f)^{-1} \nabla f_{k}^{(t)} \\
& \nabla f_{k}^{(t)}=2\left(\left\{\begin{array}{l}
\Delta N_{2} \\
\Delta M_{2}
\end{array}\right\}_{k}^{(t)}\right)^{T}\left[\begin{array}{ll}
A_{22} & B_{22} \\
B_{22} & D_{22}
\end{array}\right] d s \quad \nabla \nabla f_{k}^{(t)}=2\left[\begin{array}{ll}
A_{22} & B_{22} \\
B_{22} & D_{22}
\end{array}\right]^{T}\left[\begin{array}{ll}
A_{22} & B_{22} \\
B_{22} & D_{22}
\end{array}\right] d s
\end{aligned}
$$




\section{Nonlinear morphing model: Solution algorithm inner process}

Newton-method Up-date rule:

$\boldsymbol{\varepsilon}_{k}^{(t+1)}=-\frac{1}{\operatorname{det}}\left[\begin{array}{rr}D_{22} & -B_{22} \\ -B_{22} & A_{22}\end{array}\right]\left\{\begin{array}{l}\Delta N_{2}^{\text {ext }} \\ \Delta M_{2}^{\text {ext }}\end{array}\right\}_{k}^{t}$

converges quickly for moderate stretches however diverges at high stretches.

\section{Divergence is caused by}

the objective function deviating too much from the quadratic polynomial

estimated with Taylor-series expansion at reference point

$$
\boldsymbol{\varepsilon}_{k}^{(t+1)}=\boldsymbol{\varepsilon}_{k}^{(t)}+\Delta \boldsymbol{\varepsilon}_{k}^{(t)}
$$

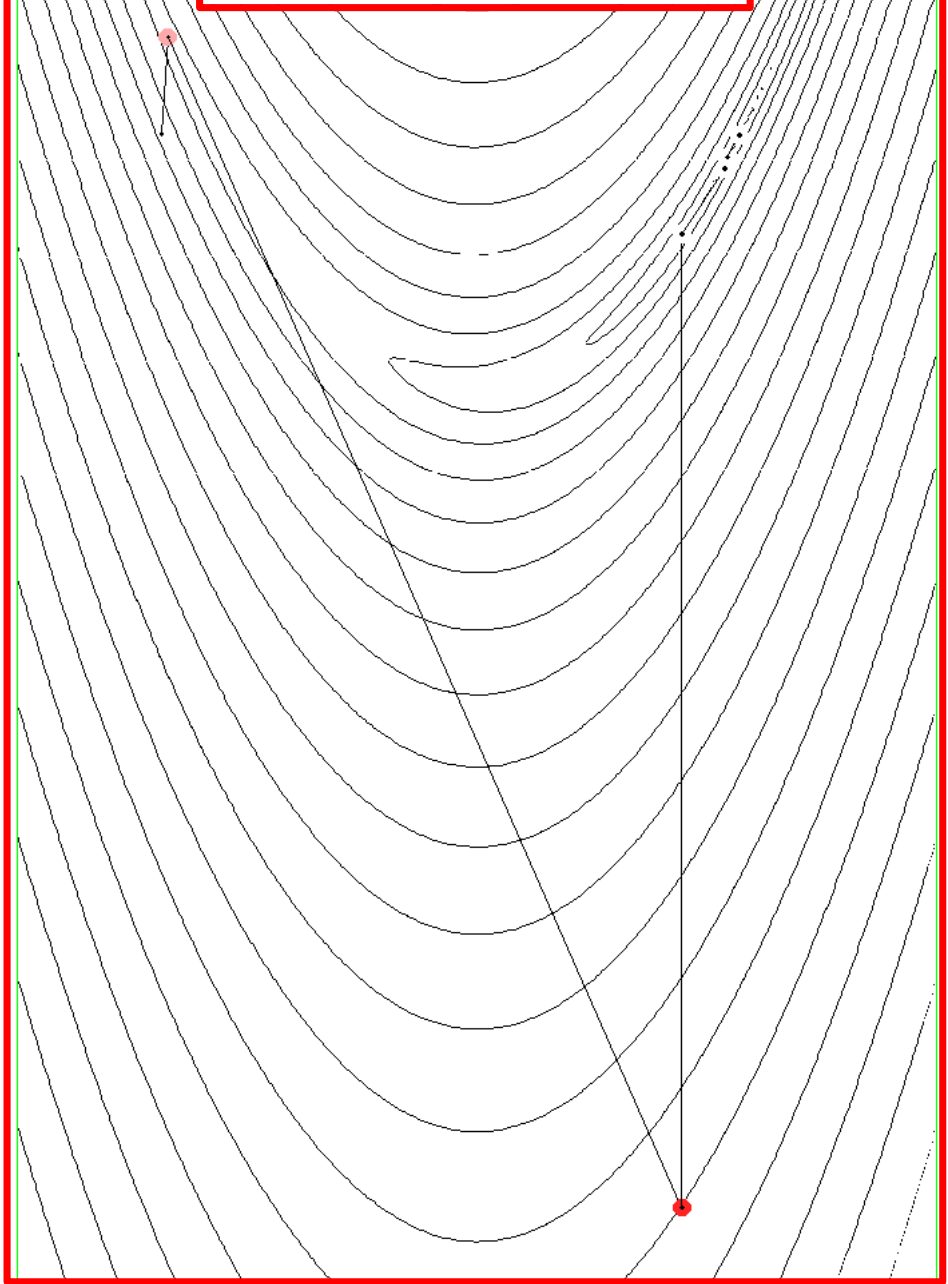

$$
\boldsymbol{\varepsilon}_{k}^{(t+1)}=\boldsymbol{\varepsilon}_{k}^{(t)}+\alpha \Delta \boldsymbol{\varepsilon}_{k}^{(t)}
$$

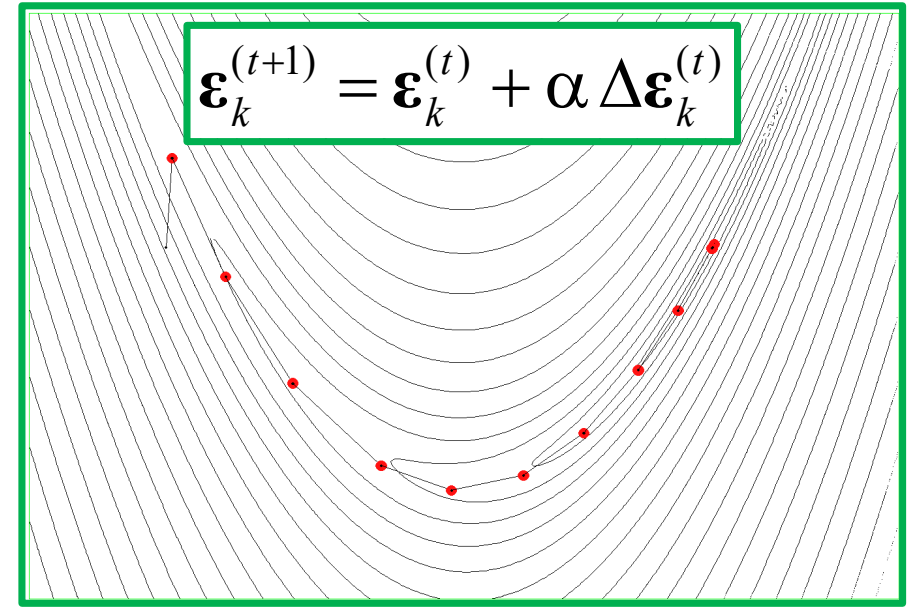

Retain convergence over full stretch range with modified Newton method, which combines minimum-point estimate with line search. 


\section{캐zürich}

\section{Nonlinear morphing model: Material and laminates}

Table 1: Relevant material properties of unidirectional T300/epoxy with fiber-volume fraction $v_{f}=0.6$. Source: DORNIER SYSTEM GmbH

\begin{tabular}{cccccc}
\hline fiber & $E_{1}$ & $E_{2}$ & $v_{12}$ & $\alpha_{1}$ & $\alpha_{2}$ \\
& \multicolumn{2}{c}{$[\mathrm{MPa}]$} & {$[-]$} & {$\left[10^{-6} / K\right]$} \\
\hline T300 & 135000 & 10000 & 0.27 & -0.6 & 30 \\
\hline
\end{tabular}




\section{ㅍzürich}

Nonlinear morphing model: Simulations

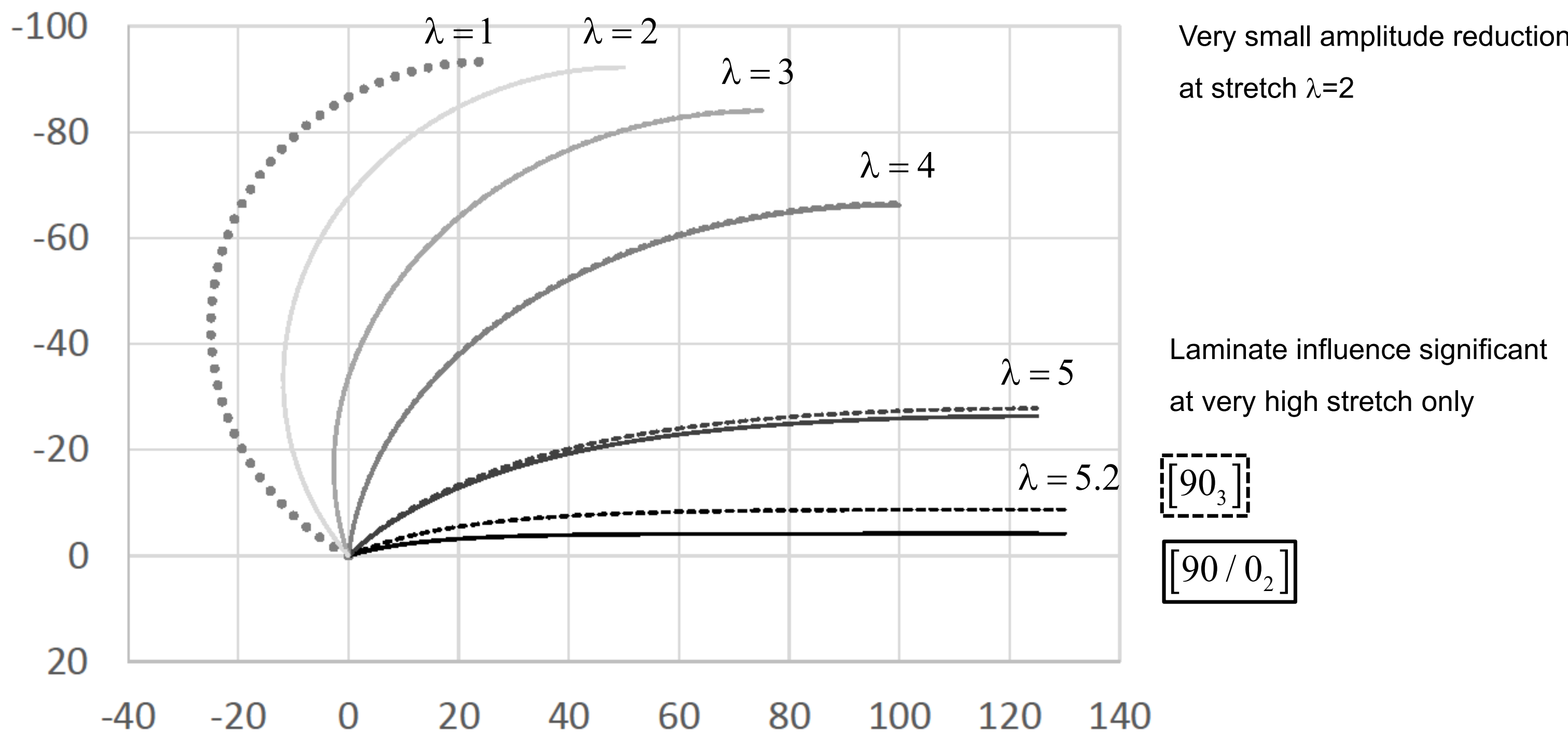


Nonlinear morphing model: Verification of deformation result by comparison with FEM

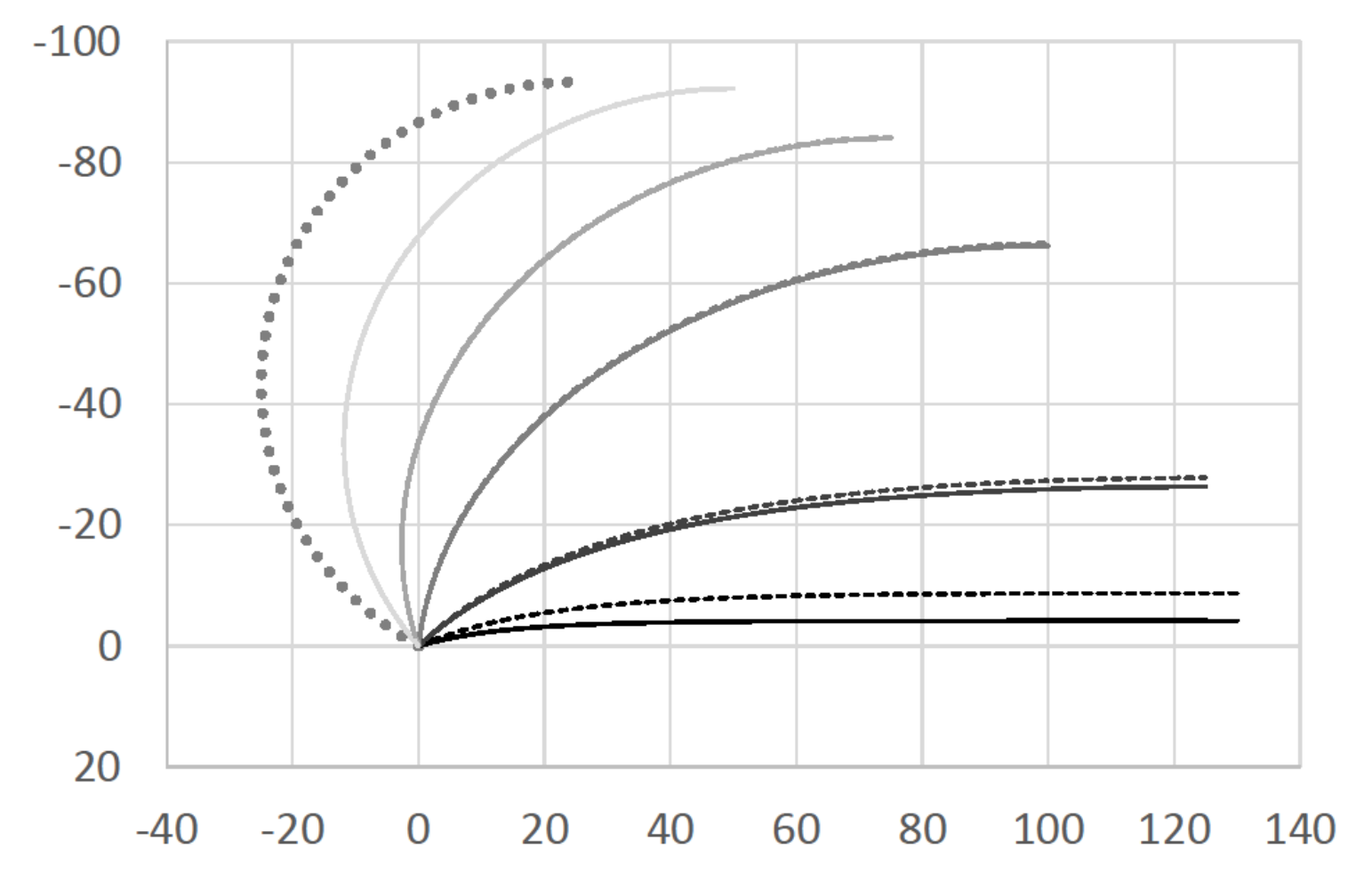

$$
\begin{aligned}
& \frac{z_{B}^{a n a}-z_{B}^{F E M}}{z_{B}^{F E M}} \approx 0.7 \% \\
& \frac{u_{z}^{a n a}-u_{z}^{F E M}}{u_{z}^{F E M}} \approx 0.07 \%
\end{aligned}
$$


Nonlinear morphing model: Influence of laminate thickness

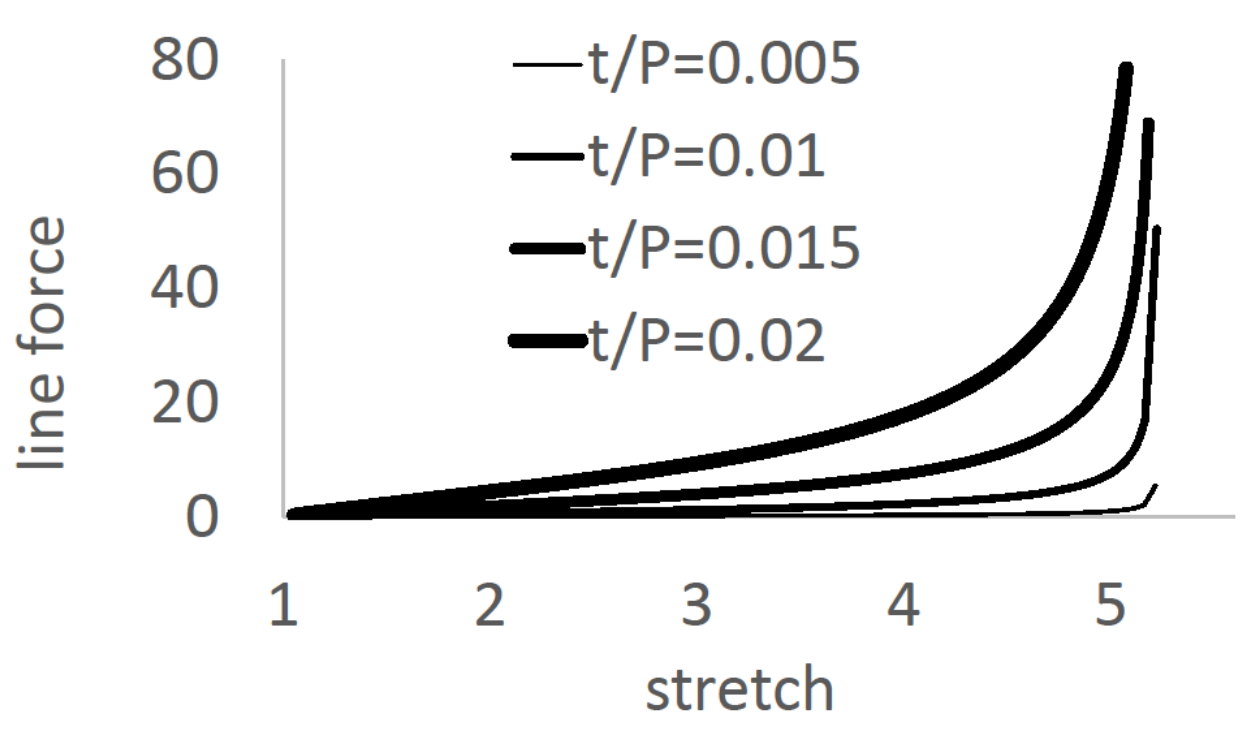

Influence of laminate thickness

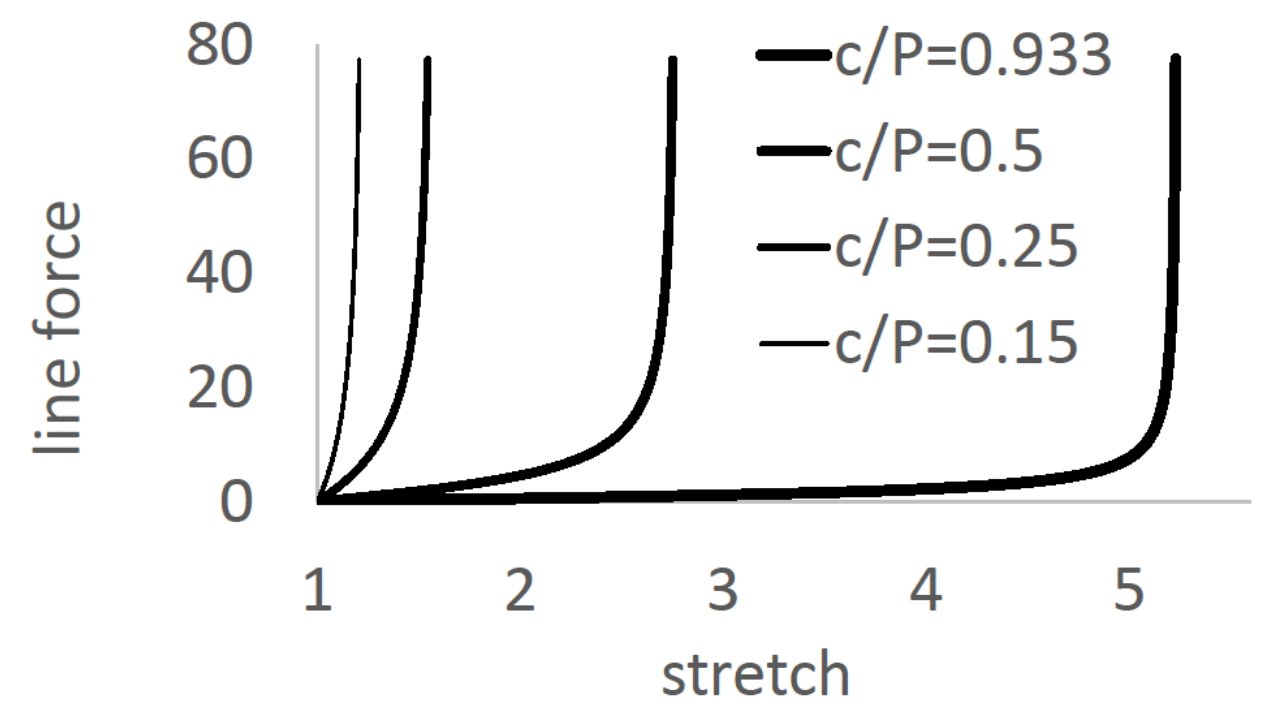

Influence of corrugation amplitude 


\section{Summary}

- Manufacturing of high-amplitude circular-sections corrugated laminates:

- is possible with exploiting thermal deformation effects in unsymmetric laminates at high curing temperatures

- thickness-to-curvature-radius ratios tend to be small

- higher ratios could be obtained with matrix systems consolidating at high temperature (PEEK: $340^{\circ} \mathrm{C}$ )

- The moldless manufacturing method naturally produces circular-sections corrugation shapes

- An analytical nonlinear morphing model has been explained.

- term «analytic» is justified with analytic form of gradient and Hesse matrix in the Newton iteration method

- Its simplicity relies on linear relation between line loads and local laminate deformations

- Results have been shown and large-deformation results have been verified by comparison with FEM 


\section{Conclusion and outlook}

- Morphing model functions up to very high stretches

- Morphing simulations show that stringer function is retained up to stretches of $\lambda=2(100 \%$ strain $)$

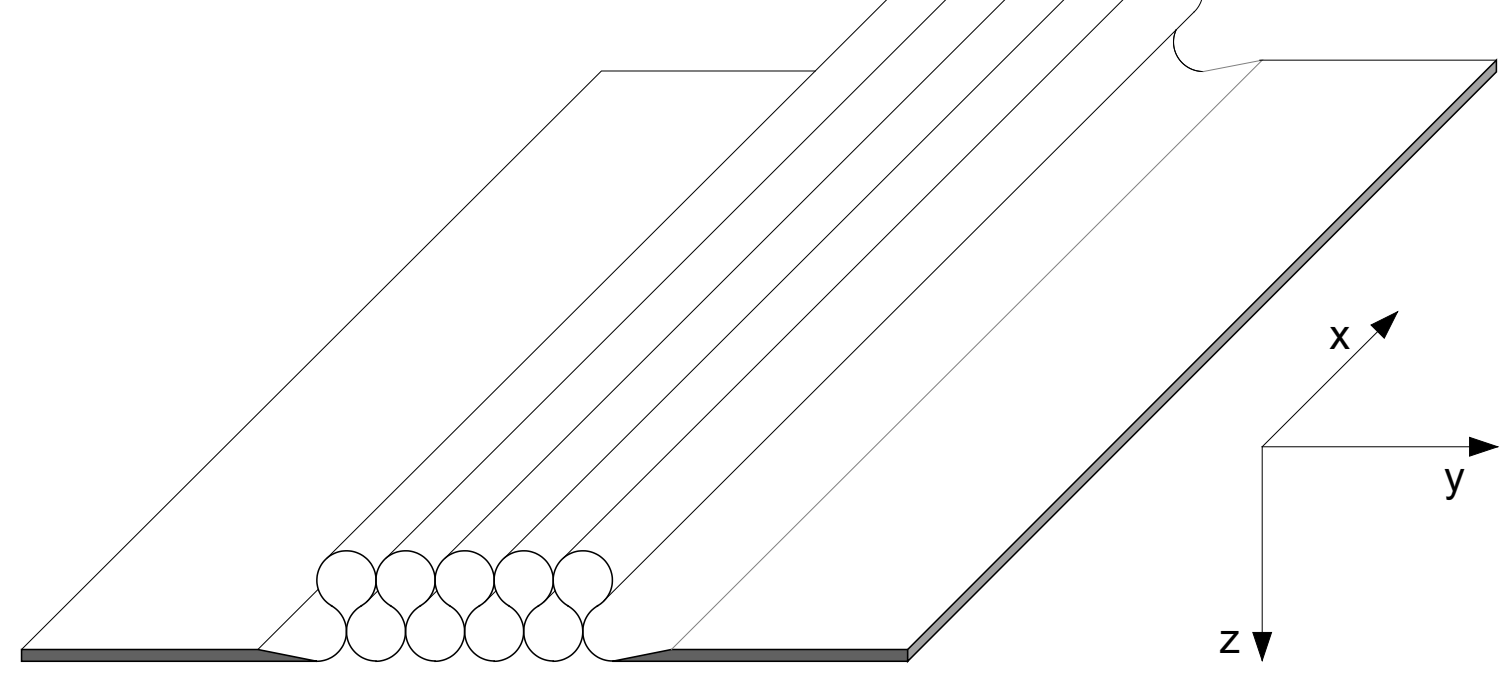

- In practical application the thickness-to-radius ratio should be higher than obtained with the demonstrator 


\section{References used for the material presented}

1. R. Jones, Mechanics of composite materials. New York: Hemisphere Publishing Corporation; 1975.

2. G. Kress, M. Winkler, Corrugated laminate homogenization model. Compos Struct, 92(3):795-810 (2010)

3. Z.X. Khoo, J.E.M. Teoh, Y. Liu, C.K.C. Chua, S. Yang, J. An, K.F. Long and W.Y. Yeong, 3D printing of smart materials: A review on recent progresses in 4D printing, Virtual and Physical Prototyping, 10(3):103-122 (2015)

4. S.V. Hoa, Factors affecting the properties of composites made by 4D printing (moldless manufacturing), Advanced Manufacturing: Polymer \& Composites Science 3(3):101-109 (2017)

5. D.T. Filipovic, G.R. Kress, Manufacturing method for high-amplitude corrugated thin-walled laminates, Composite Structures, 222:110925 (2019)

6. G. Kress, M. Winkler, Honeycomb sandwich residual stress deformation pattern, Composite Structures, 89(2): 294-302 (2009)

7. G.R. Kress, D.T. Filipovic, An analytical nonlinear morphing model for corrugated laminates, Curved and Layer. Struct., 6:56-66 (2019)

8. H. Takahashi, T. Yokozeki and Y. Hirano, Development of variable camber wing with morphing leading and trailing sections using corrugated structures, J. Int. Mat. Systems, 27(20):2827-2836 (2016)

9. J.N. Reddy, Theory and analysis of elastic plates and shells, CRC Press, Boca Baton, FI., (2007) 
Thank you for your attention - Questions ? 